\title{
THE TOPOLOGICAL SPACES THAT SUPPORT HAAR SYSTEMS
}

\author{
SCOTT MCCULLOUGH AND DANIEL WULBERT
}

\begin{abstract}
A topological space $X$ supports a nontrivial Haar system if and only if there is a continuous one-to-one map of $X$ into the unit circle. This gives an elementary proof of Mairhuber's classical theorem for compact $X$. It answers a 1960 question asked by $\mathrm{R}$. R. Phelps when $X$ is locally compact.
\end{abstract}

I. Introduction. A series of classical results around the turn of the century characterized the $n$-dimensional subspaces $H$ of continuous functions on a compact set $X$, which admit unique best approximations to each continuous function $f$. The characterizing property - now called the "Haar Condition"-is that zero is the only member of $H$ with $n$ distinct roots. (See Borel $[\mathbf{2}, 1905]$, J. W. Young [12, 1907], and Haar [6, 1918]; also see Chebyshev [3, 1859] and Bernstein $[1,1926]$.)

The existence of a nontrivial Haar space (i.e. $n \geq 2$ ) places restrictions on the compact Hausdorff space $X$. For example, it is a cute argument to show that such an $X$ cannot contain a subset that is homeomorphic to the configuration " $Y$ " in $\mathbf{R}^{2}$. Similarly $X$ cannot contain a proper subset homeomorphic to the circle. Some restrictions on $X$ were observed as early as 1913 by Haar. Mairhuber's Theorem is that $X$ supports a nontrivial Haar space if and only if $X$ is homeomorphic to a subset of the circle. This form of the result progresses from Mairhuber $[8,1956]$, Sieklucki $[11,1958]$, P. C. Curtis $[\mathbf{5}, 1959]$, and Schoenberg $[\mathbf{1 0}, 1960]$.

R. R. Phelps proved $[9,1960]$ that the Haar condition also characterizes the $n$ dimensional subspaces of $C_{0}(X)-X$ locally compact-that admitted unique best approximations. He asked there if Mairhuber's Theorem also applied to this setting.

We will show that for any topological space $X, C(X)$ contains a nontrivial Haar system if and only if there is a continuous one-to-one mapping of $X$ into the circle. This gives an easy proof of Mairhuber's Theorem. It of course also characterizes the locally compact spaces that support nontrivial Haar systems. In this setting one can ask the additional purely topological question about when such injections are homeomorphisms. They are not always homeomorphisms; but, for example, they are if the locally compact space is connected.

A curious application of the result here shows that an $n$-dimensional subspace $E$ of continuous functions on $X$ can only be defined on a one-dimensional subset of $X$ without the points becoming redundant for $E$. That is let $M \subseteq X$ be a maximal set with the property that $E$ is linearly independent on any collection of $n$ points in $M$. Then there is a continuous one-to-one map of $M$ into the circle.

Received by the editors June 8, 1984.

1980 Mathematics Subject Classification. Primary 41A45, 41A52.

Key words and phrases. Mairhuber's Theorem. 
II. Notation. An $n$-dimensional $(n \geq 2)$ linear space $H$ of continuous function is a Haar system if zero is the only function in $H$ with $n$ zeros. Throughout the paper $X$ will be a topological space that admits a Haar system $H$ and $\left\{h_{1}, \ldots, h_{n}\right\}$ will represent a basis for $H$. If $\left\{x_{i}\right\}_{i=1}^{n}$ are points in $X$ let $\operatorname{det}\left(x_{1}, \ldots, x_{n}\right)$ be the determinant of the matrix with $(i, j)$ entry equal $h_{i}\left(x_{j}\right)$. An equivalent formulation of Haar systems is that, for distinct points $\left\{x_{i}\right\}_{i=1}^{n}, \operatorname{det}\left(x_{1}, \ldots, x_{n}\right) \neq 0$ (Cheney $[4,1966$, p. 74]).

The reals are represented by $\mathbf{R}$. The continuous functions on $X$ are $C(X)$, and the sign of a real number $r$ is written $\operatorname{sgn} r$.

Special convention. We will use injection to not only mean a one-to-one mapping but to also mean that the mapping is continuous.

III. Preliminary connectivity properties. We list, for reference, some elementary topological facts.

1. LEMMA. If $W$ is connected, and $A$ and $B$ are nonempty complementary open subsets of $W-\{w\}$ (for some $w \in W$ ), then $A \cup\{w\}$ and $B \cup\{w\}$ are connected.

2. LEMMA. If $f$ is an injection of a connected set into $[0,1]$, then $f^{-1}((0,1])$ is connected.

Let

$$
Y=\{(x, 0):-1 \leq x \leq 1\} \cup\{(0, y): 0 \leq y \leq 1\} \subset \mathbf{R}^{2} .
$$

For $-1 \leq \alpha \leq 0$, put

$$
H_{\alpha}=\{(x, 0): \alpha \leq x \leq 1\}
$$

and

$$
L_{\alpha}=\{(x, 0): \alpha \leq x \leq 0\} \cup\{(0, y): 0 \leq y \leq 1\} .
$$

3. LEMMA. If $f$ is an injection of a connected set onto $Y$, then $f^{-1}\left(H_{\alpha}\right)$ and $f^{-1}\left(L_{\alpha}\right)$ are connected.

IV. Preliminary properties of Haar systems. This section contains lemmas relating Haar systems and connectivity properties of the spaces on which they are defined. The set $Y$ below is defined in the last section.

4. LEMMA. A connected subset of $X$ cannot be injected onto $Y$.

ProOF. Suppose $f$ were an injection of a connected subset of $X$ onto $Y$. Let

$$
x_{k}=f^{-1}\left(\left(\frac{1-k}{k}, 0\right)\right) \text { for } k=1,2, \ldots, n .
$$

Put

$$
y=f^{-1}((0,1)) \text { and } x=f^{-1}((1,0)) .
$$

As $z$ ranges over the connected set $f^{-1}\left(L_{0}\right), \operatorname{det}\left(z, x_{2}, x_{3}, \ldots, x_{n}\right)$ ranges over a connected nonzero set of reals. Hence

$$
\operatorname{sgn} \operatorname{det}\left(x_{1}, x_{2}, \ldots, x_{n}\right)=\operatorname{sgn} \operatorname{det}\left(y, x_{2}, \ldots, x_{n}\right) .
$$

Similarly, using the connectivity of the pre-image of $H_{-1 / 2}, L_{-1 / 2}$ and $H_{0}$, $\operatorname{sgn} \operatorname{det}\left(y, x_{2}, \ldots, x_{n}\right)=\operatorname{sgn} \operatorname{det}\left(y, x, x_{3}, \ldots, x_{n}\right)$, 


$$
\operatorname{sgn} \operatorname{det}\left(y, x, x_{3}, \ldots, x_{n}\right)=\operatorname{sgn} \operatorname{det}\left(x_{2}, x, x_{3}, \ldots, x_{n}\right),
$$

and

$$
\operatorname{sgn} \operatorname{det}\left(x_{2}, x, x_{3}, \ldots, x_{n}\right)=\operatorname{sgn} \operatorname{det}\left(x_{2}, x_{1}, x_{3}, \ldots, x_{n}\right) .
$$

However this string of equalities leads to the false statement

$$
\operatorname{sgn} \operatorname{det}\left(x_{1}, x_{2}, \ldots, x_{n}\right)=\operatorname{sgn} \operatorname{det}\left(x_{2}, x_{1}, x_{3}, \ldots, x_{n}\right) .
$$

5. LEMMA. $X$ cannot contain a proper connected subset that can be injected onto the circle.

Proof. The proof is similar in detail to the last proof, so we will only sketch the idea involved. The start of the proof is to find an even number of points $x_{1}, \ldots, x_{k}(k \leq n)$ that are carried onto the circle and additional distinct points $x_{k+1}, \ldots, x_{n}$ which are not in the proper subset. Then by continuously moving the points $x_{1}, \ldots, x_{k}$ so that their images in the circle exchange place, we see that there are distinct points in $X$ whose determinants assume each value between $\operatorname{det}\left(x_{1}, x_{2}, \ldots, x_{n}\right)$ and $\operatorname{det}\left(x_{2}, \ldots, x_{k}, x_{1}, x_{k+1}, x_{k+2}, x_{n}\right)$ and, in particular, one such set has a zero determinant.

6. LEMmA. There is a set $\left\{x_{1}, \ldots, x_{m}\right\} \subseteq X$ with $m<n$ such that $X-$ $\left\{x_{1}, \ldots, x_{m}\right\}$ is not connected.

Proof. Let $g \in H$ take both positive and negative values. The zero set of $g$ has less than $n$ points, and $g^{-1}(\mathbf{R}-\{0\})$ is disconnected.

\section{Injections of $X$ into the circle.}

7. LEMMA. If $C(X)$ contains a 2-dimensional Haar space $K$ with a nonzero function $h_{0}$, then $X$ injects into $[0,1]$.

ProOF. Replacing $K$ with $\left\{f / h_{0}: f \in K\right\}$, we have a Haar space that is spanned by $\{1, h\}$ where $h$ must be a one-to-one mapping of $X$ into the reals. Composing $h$ with a homeomorphism of $\mathbf{R}$ into $(0,1)$ gives the injection.

8. LEMMA. If $U \subseteq X$ has $n-1$ points in its complement then $U$ injects onto a subset of $[0,1]$.

PROOF. If $\left\{v_{i}\right\}_{i=1}^{n-1} \subseteq X-U$, then

$$
\left\{h \in H: h\left(v_{i}\right)=0, i=1, \ldots, n-2\right\}
$$

is a 2-dimensional Haar space on $U$ that contains the nonzero function (on $U$ ) that also vanishes at $v_{n-1}$.

Proposition. If $X$ is connected there is an injection of $X$ onto a subset of the circle.

Proof. Choose $x_{1}, \ldots, x_{m}, y$ so that $m<n, V=X-\left\{x, \ldots, x_{m}\right\}$ is connected. But $V-\{y\}$ is not connected (Lemma 6). If $A$ and $B$ are nonempty, open, complementary subsets of $V-\{y\}$, then $A \cup\{y\}$ and $B \cup\{y\}$ are then connected (Lemma 1). Since neither $A$ nor $B$ is finite, there are injections $f$ and $g$ of $A \cup\{y\}$ and $B \cup\{y\}$ respectively onto intervals whose closures are [0,1] (Lemma 8). 
We may assume that neither $f(y)$ nor $g(y)$ is equal to 1 . This in fact will force both of these to equal zero. For suppose $f(y) \neq 0$. Now

$$
C=g^{-1}([g(y), 1])
$$

is connected. We also have that $A \cup C$ is connected. Define $h$ from $A \cup C$ into $\mathbf{R}^{2}$ by

$$
h(x)= \begin{cases}(f(x), 0), & x \in A \\ (f(y), g(x)-g(y)), & x \in C .\end{cases}
$$

Since $h$ is a continuous one-to-one mapping of a connected subset of $X$ onto a homeomorphic copy of $Y$, we have contradicted Lemma 4 . We conclude that $f(y)=$ 0 . Similarly, $g(y)=0$. Hence $A$ and $B$ are connected (Lemma 2), their closures (in $X)$ are connected and these closures can be injected onto subsets of $[0,1]$ by the injections $p$ and $q$, respectively. Since these injections carry $A$ and $B$ to connected sets, $A$ and $B$ can have at most two new points in their closures. Hence, the intersection of the closures contains at most two points. If it contains two points, $X$ can be injected onto the circle; if one point, into the reals.

THEOREM. $C(X)$ contains a Haar space of dimension greater than one if and only if there is a continuous one-to-one mapping of $X$ into the unit circle.

PROOF. If $X$ has an open and closed set containing $n-1$ points and having $n-1$ points in its complement, the theorem follows from Lemma 8 . Otherwise, $X$ is composed of a connected set plus possibly a finite number of isolated points (at most $n-2)$. The connected part can be injected onto a subset of the circle. From Lemma 5 this connected part is either all of $X$ (in which case we are done), or it is a proper subset of the circle. In this case it is easy to map the remaining finite set into the circle.

COROllary (MAIRHUBER'S THEOREM). If $X$ is compact, then $C(X)$ contains a nontrivial Haar system if and only if $X$ is homeomorphic to a subset of the circle.

If $E$ is an $n$-dimensional subspace of $C(W)$ for some Hausdorff space $W$, then there are $n$ points $\left\{w_{i}\right\}$ in $W$ such that $E$ is linearly independent when viewed as functions defined only on $\left\{w_{i}\right\} . E$ is Haar if this is true for every set of $n$ noints in $W$.

We will call a subset of $W, n$-linearly independent, if $E$ is linearly independent on every subset of $n$-points. A Hausdorff maximality argument shows the existence of a maximal $n$-linearly independent set. Since $E$ is a Haar space on any $n$-linearly independent set we have

COROLlaRY. If $E$ is an n-dimensional subspace of $C(W)$ and $M \subseteq W$ is a maximal n-linearly independent set for $E$, then there is an injection of $M$ into the circle.

VI. Locally compact spaces that admit Haar systems. The following, purely topological curiosity follows from the generalized Mairhuber Theorem above. It seems likely that this result is known. 
Proposition. A locally compact, connected set $W$ that can be injected into the circle is homeomorphic to a subset of the circle.

Proof. Let $h$ be an injection of $W$ into the circle. For an $x$ in $X$, let $U$ be a neighborhood of $x$ whose compact closure $K$ is not $X$. We will show that $h(x)$ is contained in a nondegenerate arc in $h(U)$. We will assume that $h(x)=(1,0)$. Since $h(x)$ is not an isolated point, if it were not in such an arc, we can assume, without loss of generality, the existence of $0<y_{j}<u_{j}$ where $e^{i u_{j}} \in h(U), e^{i y_{j}} \notin h(U)$ and $e^{i u_{j}} \rightarrow h(x)$. Since $X$ is connected, $U_{j}=\left\{u \in U: y_{j+1}<\ln h(u)<y_{j}\right\}$ cannot be compact. We conclude that there are $b_{j} \in K-U$ with $y_{j+1}<\ln h\left(b_{j}\right) \leq y_{j}$. We arrive at the contradiction that each $b_{j} \notin U$ but $b_{j} \rightarrow x$.

Hence every $x$ has either a neighborhood base that is equivalent to open arcs

$$
\left\{e^{i r}: \ln h(x)-\varepsilon<r<\ln h(x)+\varepsilon\right\}
$$

or to half-open arcs

$$
\left\{e^{i r}: \ln h(x) \leq r<\ln h(x)+\varepsilon\right\} .
$$

If every $x$ has a neighborhood base equivalent to open arcs, then $h$ is a homeomorphism. If there is an $x$ with a neighborhood base equivalent to half open arcs, then by composing $h$ with a map of the circle onto the nonnegative reals we construct an injection $f$ of $X$ onto the nonnegative reals with $f(x)=0$. If $f$ is not a homeomorphism, there must be an $r>0$ at which $f^{-1}$ is not continuous. The neighborhood base at $f^{-1}(r)$ must be homeomorphic to half open intervals about $r$. Depending on which way the intervals face, either $f^{-1}([0, r))$ or $f^{-1}((r, \infty))$ is a nonempty open and closed subset of $X$ contradicting the connectivity of $X$.

EXAMPLE. Let $T$ be the topology on $[0,1]$ generated by designating as open, the set consisting of all the rationals as well as the usual open intervals. This space is connected, not locally compact, and hence not homeomorphic to a subset of the circle. The identity is an injection onto $[0,1]$, and so $([0,1], T)$ supports Haar spaces of all dimensions.

EXAMPLE. The discrete topology on $[0,1]$ is locally compact, not connected, not homeomorphic to a subset of $[0,1]$. However, it trivially injects onto $[0,1]$ and supports Haar spaces of all dimensions.

\section{REFERENCES}

1. S. N. Bernstein, Lecon sur les propriétés extrémales et la meilleure approximation des a fonctions analytiques d'une variable réele, Gauthier-Villars, Paris, 1926.

2. E. Borel, Lecons sur les fonctiones de variables réelles, Gauthier-Villars, Paris, 1905.

3. P. C. Chebychev, Sur les questions de minima qui se rattachent a la représentations approximative des fonctions, Oeuvres. I, 1859, pp. 273- 378.

4. E. W. Cheney, Introduction to approximation theory, McGraw-Hill, New York, 1966.

5. P. C. Curtis, N-parameter families and best approximations, Pacific J. Math. 9 (1959), 1013-1027.

6. A. Haar, Die Minkowskische Geometrie und Die Annäherung an Stetige Funktionen, Math. Ann. 78 (1918), 294-311.

7. G. W. Henderson and B. R. Ummel, The nonexistence of complex Haar systems of nomplanar locally connected spaces, Proc. Amer. Math. Soc. 39 (1973), 640-641.

8. J. C. Mairhuber, On Haar's theorem concerning Chebychev approximation problems having unique solutions, Proc. Amer. Math. Soc. 7 (1956), 609-615.

9. R. R. Phelps, Uniqueness of Hahn Banach extensions and unique best approximations, Trans. Amer. Math. Soc. 95 (1960), 238-255. 
10. I. J. Schoenberg, On the question of unicity in the theory of best approximation, New York Acad. Sci. 86 (1960), 682-692.

11. K. Sieklucki, Topological properties of sets admitting Tchebycheff systems, Bull. Acad. Polon. Sci. Sér. Sci. Math. 6 (1958), 603-606.

12. J. W. Young, General theory of approximation by functions involving a given number of parameters, Trans. Amer. Math. Soc. 8 (1907), 331-344.

Department of Mathematics, University of CAlifornia, SAN Diego, La Jolla, CALIFORNIA 92093 